\title{
Formulation of the stability margins clearance criterion as a convex optimization problem
}

Christos Papageorgiou, Rikard Falkeborn, Anders Hansson

Division of Automatic Control

E-mail: papageorgiou@isy.liu.se, falkeborn@isy.liu.se, hansson@isy.liu.se

19th October 2009

Report no.: LiTH-ISY-R-2920

Accepted for publication in 6th IFAC Symposium on Robust Control Design

Address:

Department of Electrical Engineering

Linköpings universitet

SE-581 83 Linköping, Sweden

WWW: http://wWw. control.isy.liu.se

AUTOMATIC CONTROL

REGLERTEKNIK

LINKÖPINGS UNIVERSITET

Technical reports from the Automatic Control group in Linköping are available from http://www . control.isy.liu.se/publications. 


\begin{abstract}
This paper presents the formulation of a flight clearance criterion as a convex optimization problem. The criterion is the stability margins criterion which is specified as an allowable phase and gain margin of a certain loop transfer function. The satisfaction of the criterion amounts to the Nichols plot of the loop transfer function being outside a specified trapezoidal region. It was shown previously that the exclusion condition from an ellipsoidal region is implied by using the generalized stability margin $b_{P C}$ and its calculation was performed frequency-wise by solving a sequence of convex optimization problems. In this paper we formulate the calculation of a lower bound on $b_{P C}$ as a convex optimization problem using Integral Quadratic Constraints (IQCs) and avoid the gridding procedure in the frequency domain. Furthermore, we formulate the problem of obtaining a lower bound on the perturbed stability margin, which is defined as the worst-case $b_{P C}$ over variations in real uncertain parameters.
\end{abstract}

Keywords: Robust Stability and Performance, Computational Methods, LMI and Convex Optimization 


\title{
Formulation of the stability margins clearance criterion as a convex optimization problem *
}

\author{
Christos Papageorgiou ${ }^{* *}$ Rikard Falkeborn ${ }^{* * *}$ Anders Hansson ${ }^{* * * *}$ \\ Automatic Control, University of Linköping 58183 LINKÖPING, SWEDEN, \\ ** email:papageorgiou@isy.liu.se \\ *** email:falkeborn@isy.liu.se \\ ****email:hansson@isy.liu.se
}

\begin{abstract}
This paper presents the formulation of a flight clearance criterion as a convex optimization problem. The criterion is the stability margins criterion which is specified as an allowable phase and gain margin of a certain loop transfer function. The satisfaction of the criterion amounts to the Nichols plot of the loop transfer function being outside a specified trapezoidal region. It was shown previously that the exclusion condition from an ellipsoidal region is implied by using the generalized stability margin $b_{P C}$ and its calculation was performed frequency-wise by solving a sequence of convex optimization problems. In this paper we formulate the calculation of a lower bound on $b_{P C}$ as a convex optimization problem using Integral Quadratic Constraints (IQCs) and avoid the gridding procedure in the frequency domain. Furthermore, we formulate the problem of obtaining a lower bound on the perturbed stability margin, which is defined as the worst-case $b_{P C}$ over variations in real uncertain parameters.
\end{abstract}

\section{INTRODUCTION}

The clearance of a flight control law is defined as the certification procedure which proves that the control law will satisfy the clearance criteria for a given flight envelope and in the presence of failure conditions and uncertainty ([4]). The procedure consists of two tasks: the verification of 'safe' regions in the flight envelope and the uncertain parameter space and the detection of a combination of uncertain parameters and/or pilot inputs and/or flight conditions that will result in a clearance criterion being violated. The clearance criteria are measures to quantify stability and performance (flying and handling qualities) under all possible flying conditions. The flight envelope is defined as the set of possible flying conditions and is characterized by flight parameters such as speed, altitude and angle of attack. The flight control law is often scheduled on the flight envelope parameters to achieve better overall performance. The uncertainty is defined as the set of uncertain parameters whose values are not known but they have known ranges of variation and bounded rates of variation. Common uncertain parameters are the position of the center of gravity, the mass and inertia coefficients and the aerodynamic coefficients of the aircraft.

The industrial clearance procedure is performed in various stages, starting from a computer-based analysis by employing a model of the aircraft and the control law, then moving on to Hardware-In-The-Loop tests, real-time simulator tests and finally flight testing. In our work we concentrate on computerbased or model-based analysis and we attempt to use optimization methods in order to obtain more reliable results. The current model-based analysis involves mainly linear analysis techniques such as the evaluation of phase and gain margins ([1]), the calculation of closed-loop eigenvalues and the examination of the frequency responses of selected input-output channels. The procedure involves the trimming and linearization of the

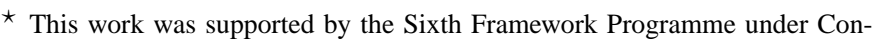
tract AST5-CT-2006-030768-COFCLUO (Clearance of Flight Control Laws Using Optimisation).
}

aircraft model and the controller on a fine grid over the flight envelope and the uncertain parameter space. If there exists a large number of flight parameters and uncertain parameters, this creates a huge number of linear closed-loop models that need to be tested for clearance. Due to the gridding involved, this approach is not reliable and an attempt to improve reliability by using a finer grid requires excessive computational effort.

The first step towards implementing a flight clearance task as a convex optimization problem is to use ideas from robust control theory ([16]) and formulate the clearance task either as a robust stability or a robust performance problem with respect to variations in the uncertain parameters and the flight envelope parameters. Powerful paradigms of robust control theory such as the Linear Fractional Transformation (LFT) paradigm can be used to avoid the gridding of the flight envelope and the uncertain parameter space. Using this approach, the uncertain closed-loop system is embedded with some conservatism in an LFT representation, i.e. an interconnection of a linear system with an uncertain $\Delta$-block that contains information about the uncertain parameters and the flight envelope parameters. There is a trade-off between the complexity of the LFT and the associated conservatism in embedding the actual system dynamics ([6]). The LFT paradigm can be used to model both linear and nonlinear uncertain systems, although in the latter case additional parameters are introduced in the $\Delta$-block that describe the nonlinearity of the model. After describing the uncertain aircraft model as an LFT, the clearance task can be seen as a solution of a robustness analysis problem. Indeed, the natural approach would be to use the popular $\mu$-analysis technique in the case of robust stability analysis with respect to real uncertain parameters and/or unmodelled dynamics ([3]). Employing robust control theory gives superior reliability to the conventional gridding procedure and at the same time it avoids the solution of the clearance task at each grid point. There is a catch with the LFT approach though. If the generated LFT is required to capture the closed-loop system dynamics over a larger flight envelope and uncertain parameter set and with 
less conservatism, the size of the LFT increases in a way that it prohibits the use of the conventional techniques for the solution of the robustness analysis problem. This fact has long been recognized and considerable research has been conducted in the area of generating minimum size LFT models ([8]).

A solution to a lot of robustness analysis problems such as robust stability with respect to uncertain parameters or robust $\mathscr{H}_{2}$ and $\mathscr{H}_{\infty}$ minimization can be obtained using convex optimization and in particular Semidefinite Programming (SDP) which is the solution of an optimization problem with the cost function being a linear function of the decision variables and the constraints being Linear Matrix Inequalities (LMIs) ([2]). The formulation of a robustness analysis problem as an SDP results by searching for Lyapunov functions (either constant or parameter-dependent) to prove stability and performance or by assessing robust stability using the theory of Integral Quadratic Constraints ([10]) in conjunction with the KYP Lemma. These techniques have been applied, mainly on a research level, for the analysis of various flight clearance problems both stability and performance related ([11]).

In our work we have used a tool from robust control theory in order to solve the stability margins flight clearance problem. This tool is the generalized stability margin $b_{P C}$ which is associated with the $\mathscr{H}_{\infty}$-loopshaping methodology ([9]) for designing robust controllers. It is a measure of robustness with respect to uncertainty in the coprime factors of a system, but most importantly, it has been shown to have a direct connection to the Nichols exclusion criteria used for the evaluation of stability margins ([5]). Previously, the $b_{P C}$ was used in a GARTEUR project for the evaluation of the stability margins and its calculation was performed frequency-wise by solving an SDP at each frequency over a selected grid of frequencies ([12]). In our approach we exploit the theory of IQCs to formulate the problem of calculating a lower bound on $b_{P C}$ without resorting to frequency gridding. The frequency gridding may give misleading results if a wrong frequency grid is used in the analysis of aircraft with lightly damped flexible modes.

\section{SPECIFYING NICHOLS EXCLUSION REGIONS USING $b_{P C}$}

In this section we show how the generalized stability margin can be used to analyze the stability of closed-loop systems and how the results can be related to exclusion regions in the Nichols diagrams which is an industry standard for the clearance of flight control laws.

Given the closed-loop system in Fig. 1 consisting of a nominal plant $P$ and a controller $C$ (designed with any control design method), apply stable, minimum-phase weights in such a way so that the loop transfer matrix remains unchanged. Therefore, the weights are applied at the inputs and outputs of the plant with their inverses applied at the inputs and outputs of the controller as shown in Fig. 1. The weighted generalized stability margin is defined as,

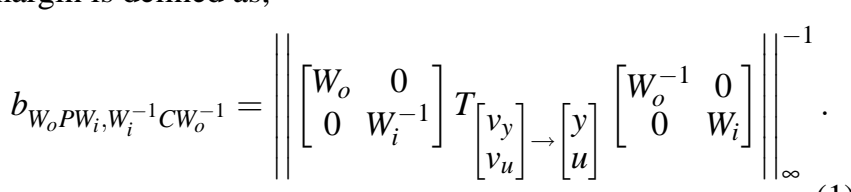

Given the plant transfer matrix $P$ and the controller transfer matrix $C$, we can calculate the closed-loop transfer matrix as,

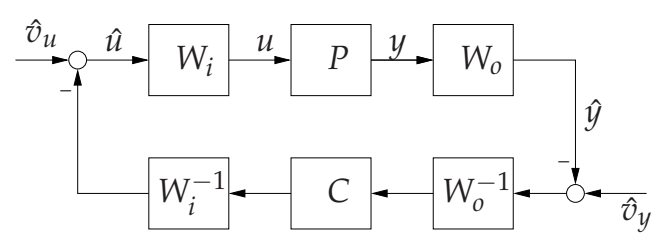

Fig. 1. The placement of weights for the calculation of the weighted generalized stability margin.

$$
T_{c l}(s):=T_{\left[\begin{array}{l}
v_{y} \\
v_{u}
\end{array}\right] \rightarrow\left[\begin{array}{l}
y \\
u
\end{array}\right]}=\left[\begin{array}{c}
P \\
I
\end{array}\right](I-C P)^{-1}\left[\begin{array}{ll}
-C & I
\end{array}\right] .
$$

This closed-loop transfer matrix corresponds to the transfer matrix from external disturbances $\left(v_{u}, v_{y}\right)$ injected at the inputs and outputs of the plant to the inputs and outputs $(u, y)$ of the plant. Its stability is equivalent to the internal stability of the closed-loop system and it contains all the important closedloop transfer functions in terms of characterizing performance, like the sensitivity function $S$, the complementary sensitivity function $T$, the control sensitivity function and the plant sensitivity function. The definition of $b_{W_{o} P W_{i}, W_{i}^{-1} C W_{o}^{-1}}$ as a stability margin is given in [14] and it can be shown that it is equal to the maximum size of the coprime factor uncertainty of the weighted plant for which stability is guaranteed.

In the analysis of the closed-loop system, the objective is to construct weights $W_{o}$ and $W_{i}$ to maximize the stability margin, or minimize the weighted $\mathscr{H}_{\infty}$ norm. Once we perform the optimization $b_{P C}=\max _{W_{o}, W_{i}} b_{W_{o} P W_{i}, W_{i}^{-1} C W_{o}^{-1}}$, we can relate the optimal value to the robust stability of the closed-loop system with respect to input and output independent and simultaneous multiplicative perturbations applied at the plant using the following theorem.

Theorem 2.1. ([5]). Let $\Delta_{1}$ and $\Delta_{2}$ be complex diagonal matrices which perturb a nominal plant $P$ to $P_{\Delta}=\left(I+\Delta_{1}\right) P(I-$ $\left.\Delta_{2}\right)^{-1}$. If the weighted stability margin satisfies,

$$
b_{W_{o} P W_{i}, W_{i}^{-1} C W_{o}^{-1}} \geq \beta
$$

for any diagonal input and output analysis weights, $W_{i}, W_{o}$, then $\left[P_{\Delta}, C\right]$ is stable for any perturbations satisfying $\left\|\Delta_{2}\right\|_{\infty}<\beta$ and $\left\|\Delta_{1}\right\|_{\infty}<\beta$.

Again from the above theorem we see the motivation for maximizing $b_{W_{o} P W_{i}, W_{i}^{-1} C W_{o}^{-1}}$ (or its lower bound $\beta$ ) over diagonal weights since we can guarantee the robust stability of the closed-loop system with a perturbed plant $P_{\Delta}$ for increasing amount of uncertainty. The final step to link $b_{P C}$ to the Nichols stability margins is the characterization of the perturbed plant $P_{\Delta},\left(\left\|\Delta_{1}\right\|_{\infty},\left\|\Delta_{2}\right\|_{\infty}<\beta\right)$ using multiplicative input-output gain-phase perturbations. Re-write the perturbed plant as,

$$
P_{\Delta}=\frac{1}{\sqrt{1-\beta^{2}}}\left(I+\Delta_{1}\right) P\left(I-\Delta_{2}\right)^{-1} \sqrt{1-\beta^{2}} .
$$

For $\beta<1$ (which is the case since $0 \leq b_{P C} \leq 1$ ), the sets $\left\{\frac{1+\delta_{1}}{\sqrt{1-\beta^{2}}}:\left|\delta_{1}\right|<\beta\right\}$ and $\left\{\frac{\sqrt{1-\beta^{2}}}{1-\delta_{2}}:\left|\delta_{2}\right|<\beta\right\}$ are identical, therefore the closed-loop system is robust with respect to simultaneous and independent multiplicative perturbations as shown in Fig. 2. The set $\left\{\frac{1+\delta}{\sqrt{1-\beta^{2}}}:|\delta|<\beta\right\}$ describes an ellipse if we consider the logarithm of the magnitude against the phase of the complex number. An example for $\beta=0.3$ is shown in 


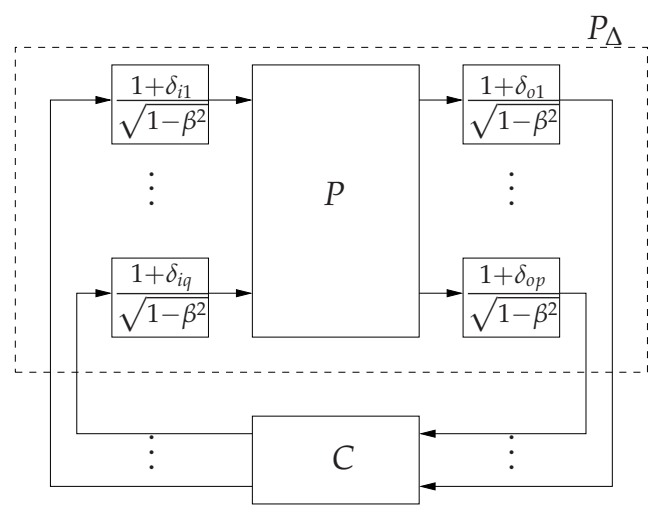

Fig. 2. The interconnection of the multiplicatively perturbed

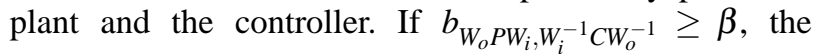
closed-loop system can tolerate the multiplicative perturbations shown in the figure for any $|\delta|<\beta$.

Fig. 3 plotted in red. In the SISO case the set is actually described by $\left\{\frac{(1+\delta)^{2}}{1-\beta^{2}}:|\delta|<\beta\right\}$ since we can merge the input and output perturbation. This gives an ellipse twice as big as the one obtained in the general MIMO case. It is important to remember

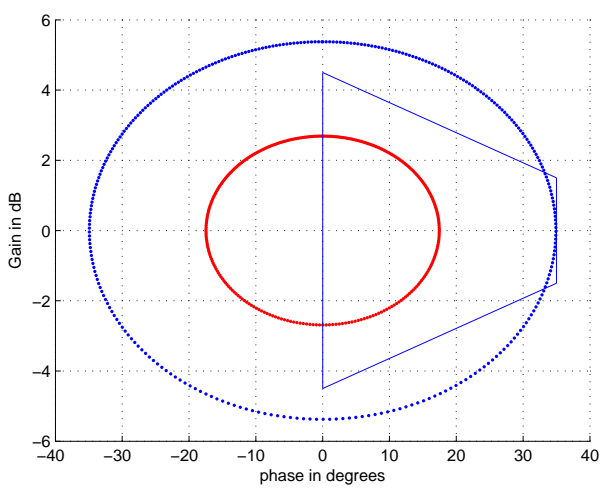

Fig. 3. The region described by the multiplicative perturbation set for $\beta=0.3$ both in the SISO (larger ellipse) and MIMO (smaller ellipse) cases.

that although in the MIMO case the perturbation is smaller, it can be tolerated simultaneously at both the input and output of a certain channel. If in the MIMO case we wish to consider a perturbation at a single loop then this is given by the same perturbation as in the SISO case [5]. The trapezoidal region specifies the normal SISO gain and phase margin requirements from an industrial point of view. It is easy to see that a value of $\beta>0.3$ will guarantee the clearance of the gain and phase margin criterion.

\section{CALCULATION OF A LOWER BOUND ON $B_{P C}$}

In this section we propose an optimization method to calculate a lower bound on the value of $b_{P C}$. The lower bound is obtained using an IQC analysis of a certain closed-loop interconnection.

In order to calculate $b_{P C}^{-1}$ we must perform the following optimization,

$$
b_{P C}^{-1}=\min _{W(s) \operatorname{diag}}\left\|W(s) T_{c l}(s) W^{-1}(s)\right\|_{\infty},
$$

where $W(s)=\operatorname{diag}\left(W_{o}(s), W_{i}^{-1}(s)\right)$. Substituting for the definition of the $\mathscr{H}_{\infty}$ norm we have that,

$$
\begin{aligned}
b_{P C}^{-1} & =\min _{W(j \omega) \operatorname{diag}} \max _{\omega} \bar{\sigma}\left(W(j \omega) T_{c l}(j \omega) W^{-1}(j \omega)\right) \\
& =\max _{\omega} \min _{\operatorname{diag} W>0} \bar{\sigma}\left(W T_{c l}(j \omega) W^{-1}\right) .
\end{aligned}
$$

The second equality states that the optimal weight can be found by frequency-wise optimization over its magnitude and then uniquely reconstruct its phase since it is minimum-phase.

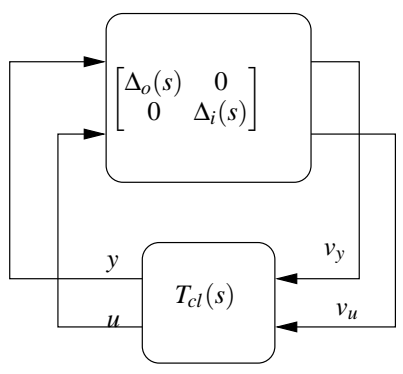

Fig. 4. Robustness analysis with respect to a structured diagonal uncertainty.

The value of $b_{P C}$ obtained by frequency-wise optimization must be treated with caution in the case of analysis of closed-loop systems with lightly-damped flexible modes since the choice of the frequency grid may affect the result. The effect on the choice of the frequency grid maybe assessed by employing the lower bound on $b_{P C}$ which can be obtained via an optimization problem that does not require frequency-wise optimization.

For the lower bound calculation, consider the problem of analyzing the stability of the closed-loop system in Fig. 4 using IQCs. The first step is to use IQCs to characterize the uncertainty. Assume that the structured uncertainty $\Delta=$ $\operatorname{diag}\left(\Delta_{o}(s), \Delta_{i}(s)\right)$ satisfying $\left\|\Delta_{i}(s)\right\|_{\infty} \leq \gamma,\left\|\Delta_{o}(s)\right\|_{\infty} \leq \gamma$ satisfies the following IQC,

$$
\int_{-\infty}^{\infty}\left[\begin{array}{l}
\hat{y}(j \omega) \\
\hat{u}(j \omega) \\
\hat{v}_{y}(j \omega) \\
\hat{v}_{u}(j \omega)
\end{array}\right]^{*}\left[\begin{array}{cc}
\Pi(j \omega) & 0 \\
0 & -\frac{1}{\gamma^{2}} \Pi(j \omega)
\end{array}\right]\left[\begin{array}{l}
\hat{y}(j \omega) \\
\hat{u}(j \omega) \\
\hat{v}_{y}(j \omega) \\
\hat{v}_{u}(j \omega)
\end{array}\right] d \omega \geq 0
$$

where,

$$
\Pi(j \omega)=\operatorname{diag}\left(\Pi_{o}(j \omega), \Pi_{i}(j \omega)\right), \Pi(j \omega)=\Pi(j \omega)^{*} \geq 0 .(8)
$$

A sufficient condition for the closed-loop stability of the interconnection in Fig. 4 is the existence of a multiplier $\Pi(j \omega)$ such that,

$$
\begin{aligned}
& {\left[\begin{array}{c}
T_{c l}(j \omega) \\
I
\end{array}\right]^{*}\left[\begin{array}{cc}
\Pi(j \omega) & 0 \\
0 & \left.-\frac{1}{\gamma^{2}} \Pi_{(} j \omega\right)
\end{array}\right]\left[\begin{array}{c}
T_{c l}(j \omega) \\
I
\end{array}\right]<0 \forall \omega \Leftrightarrow } \\
\Leftrightarrow & T_{c l}^{*}(j \omega) \Pi(j \omega) T_{c l}(j \omega)-\frac{1}{\gamma^{2}} \Pi(j \omega)<0 \forall \omega .
\end{aligned}
$$

Given that we can factorize $\Pi(j \omega)=W^{*}(j \omega) W(j \omega)$, we substitute in the previous inequality and also right-multiply by $W^{-1}(j \omega)$ and left-multiply by $W^{-*}(j \omega)$ to obtain the equivalent inequality,

$$
(X(j \omega))^{*}(X(j \omega))-\frac{1}{\gamma^{2}} I<0 \forall \omega,
$$

with

$$
X(j \omega)=W(j \omega) T_{c l}(j \omega) W^{-1}(j \omega) .
$$

The above inequality is equivalent to,

$$
\sup _{\omega} \bar{\sigma}(X(j \omega))<\frac{1}{\gamma} \Leftrightarrow\left\|W(s) T_{c l}(s) W^{-1}(s)\right\|_{\infty}<\frac{1}{\gamma} .
$$


We know that $b_{P C}^{-1}=\min _{\operatorname{diag} W(s)}\left\|W(s) T_{c l}(s) W^{-1}(s)\right\|_{\infty}$ and we have found a candidate diagonal weight that satisfies an upper bound on the $\mathscr{H}_{\infty}$ norm of the weighted closed-loop transfer matrix. This implies that $b_{P C}>\gamma$. Therefore, checking the robust stability of the closed-loop system in Fig. 4 for increasing values of the uncertainty size $\gamma$ will provide successively improved lower bounds on $b_{P C}$. The optimization problem for obtaining the lower bound is:

$$
\begin{aligned}
& \min _{\Pi(j \omega)} \frac{1}{\gamma^{2}} \\
& \text { subject to (9) }
\end{aligned}
$$

Using a bisection algorithm on $\frac{1}{\gamma^{2}}$ and using appropriate statespace representations for $\Pi(j \omega)$ and $T_{c l}(j \omega)$, we can apply the KYP-Lemma on condition (9), to transform it into an LMI problem. It is also possible to avoid the step of transforming the frequency-domain inequality (9) into an LMI problem by using the IQC-toolbox which automates this step for various types of uncertainties.

\section{FORMULATION OF THE PERTURBED STABILITY MARGIN PROBLEM}

As mentioned previously, we are required to estimate not only the nominal stability margins but also the perturbed stability margins in the presence of real, parametric uncertainty. This parametric uncertainty is either due to uncertain physical parameters or due to flight envelope parameters. Assume that the set of the parametric uncertainty is given by,

$\Delta_{u}=\left\{\Delta_{u}: \Delta_{u}=\operatorname{diag}\left(\delta_{1} I_{k_{1}}, \ldots, \delta_{m} I_{k_{m}}\right), \delta_{i} \in \mathbb{R},\left|\delta_{i}\right| \leq \varepsilon\right\}$.

The problem of calculating the worst-case stability margin $b_{P C, W C}^{-1}$ for a given uncertainty size $0<\varepsilon \leq 1$ can be formulated as follows with reference to Fig. 5.

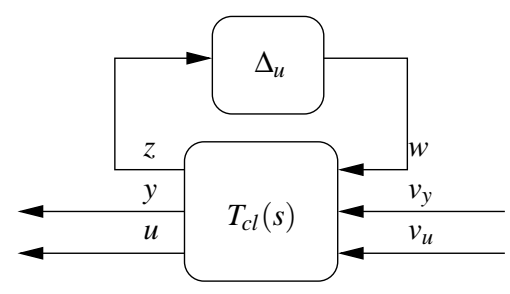

Fig. 5. The closed-loop system for the formulation of the perturbed stability margin problem. Note that the transfer matrix $T_{c l}(s)$ is not the same as the one used for the calculation of the nominal stability margin.

$b_{P C, W C}^{-1}(\varepsilon):=\min _{W(s) \operatorname{diag}} \sup _{\Delta_{u} \in \Delta_{u}}\left\|W(s) \mathscr{F}_{u}\left(T_{c l}(s), \Delta_{u}\right) W^{-1}(s)\right\|_{\infty}$,

where $\mathscr{F}_{u}\left(T_{c l}(s), \Delta_{u}\right)$ is the perturbed closed-loop system expressed as an upper LFT of the nominal closed-loop transfer matrix $T_{c l}(s)$ with respect to the structured uncertainty $\Delta_{u}$. Clearly, as $\varepsilon \rightarrow 0$ we have $\Delta_{u} \rightarrow 0$ and we approach the nominal stability margin. The idea here is to gradually increase $\varepsilon$ to 1 (the normalization of $\Delta_{u}$ captures the physical parameter variations) and investigate the possible degradation in the stability margin value as a function of the uncertainty size.

A lower bound on the perturbed stability margin can be obtained by utilizing the robust performance analysis result of the IQC theory and applying it for the case where the desired performance is expressed as a weighted $\mathscr{H}_{\infty}$ gain. Assume that the parametric uncertainty satisfies the IQC given by,

$$
\int_{\infty}^{\infty}\left[\begin{array}{c}
\hat{z}(j \omega) \\
\hat{w}(j \omega)
\end{array}\right]^{*}\left[\begin{array}{ll}
\Pi_{11}(j \omega) & \Pi_{12}(j \omega) \\
\Pi_{12}^{*}(j \omega) & \Pi_{22}(j \omega)
\end{array}\right]\left[\begin{array}{c}
\hat{z}(j \omega) \\
\hat{w}(j \omega)
\end{array}\right] d \omega \geq 0 .
$$

Theorem 4.1. (Weighted robust $L_{2}$-gain performance). Assume that any uncertainty $\Delta_{u} \in \Delta_{u}$ satisfies the IQC given in (15). If there exists a multiplier $\Pi(j \omega)$ and a $\gamma$ such that the following frequency-domain inequality holds

$$
\left[\begin{array}{c}
T_{c l}(j \omega) \\
I
\end{array}\right]^{*}\left[\begin{array}{cccc}
\Pi_{11}(j \omega) & 0 & \Pi_{12}(j \omega) & 0 \\
0 & \Pi(j \omega) & 0 & 0 \\
\Pi_{12}^{*}(j \omega) & 0 & \Pi_{22}(j \omega) & 0 \\
0 & 0 & 0 & -\frac{1}{\gamma^{2}} \Pi(j \omega)
\end{array}\right]\left[\begin{array}{c}
T_{c l}(j \omega) \\
I
\end{array}\right]<0 \quad \forall \omega
$$

then

$$
\begin{array}{r}
\left\|W(s) \mathscr{F}_{u}\left(T_{c l}(s), \Delta_{u}\right) W^{-1}(s)\right\|_{\infty} \leq \frac{1}{\gamma} \forall \Delta_{u} \in \Delta_{u} \\
\text { with } \Pi(j \omega)=W^{*}(j \omega) W(j \omega) .
\end{array}
$$

The satisfaction of the inequality in (17) implies the following:

$$
b_{P C, W C}(\varepsilon) \geq \gamma \text {. }
$$

Proof: Assume that there exists a multiplier $\Pi(j \omega)$ that satisfies the FDI in (16) for a chosen value of $\frac{1}{\gamma^{2}}$. If we post-multiply the FDI by the matrix,

$$
\hat{W}(j \omega)=\left[\begin{array}{cc}
I & 0 \\
0 & W(j \omega)^{-1}
\end{array}\right]
$$

and pre-multiply by its conjugate, then it will still be valid. The next step is to use the factorization of the multiplier $\Pi(j \omega)$ in terms of $W(j \omega)$ and factorize the central matrix in the FDI. This will result in the place of $\Pi(j \omega)$ in the central matrix to have $I$ instead and to post-multiply the new central matrix with the factor,

$$
\left[\begin{array}{cc}
\hat{W}(j \omega) & 0 \\
0 & \hat{W}(j \omega)
\end{array}\right]
$$

and pre-multiply by its conjugate. We can then form,

$$
\left[\begin{array}{c}
\hat{T}_{c l}(j \omega) \\
I
\end{array}\right]=\left[\begin{array}{cc}
\hat{W}^{-1}(j \omega) & 0 \\
0 & \hat{W}^{-1}(j \omega)
\end{array}\right]\left[\begin{array}{c}
T_{c l}(j \omega) \\
I
\end{array}\right] \hat{W}(j \omega) .
$$

If we adopt a $2 \times 2$ partitioning of the closed-loop transfer matrix $T_{c l}$ in accordance with the separation between the performance signals and the uncertainty signals in Fig. 5, we can also partition $\hat{T}_{c l}(j \omega)$ as,

$$
\hat{T_{c l}}(j \omega)=\left[\begin{array}{cc}
I & 0 \\
0 & W
\end{array}\right]\left[\begin{array}{ll}
T_{c l_{11}}(j \omega) & T_{c l_{12}}(j \omega) \\
T_{c l_{21}}(j \omega) & T_{c l_{22}}(j \omega)
\end{array}\right]\left[\begin{array}{cc}
I & 0 \\
0 & W^{-1}
\end{array}\right] .
$$

The FDI in (16) is now transformed to,

$$
\left[\begin{array}{c}
\hat{T}_{c l}(j \omega) \\
I
\end{array}\right]^{*}\left[\begin{array}{clcc}
\Pi_{11}(j \omega) & 0 & \Pi_{12}(j \omega) & 0 \\
0 & I & 0 & 0 \\
\Pi_{12}^{*}(j \omega) & 0 & \Pi_{22}(j \omega) & 0 \\
0 & 0 & 0 & -\frac{1}{\gamma^{2}} I
\end{array}\right]\left[\begin{array}{c}
\hat{T}_{c l}(j \omega) \\
I
\end{array}\right]<0 \quad \forall \omega
$$

The validity of the FDI in (23) implies the robust $L_{2}$-gain performance condition (or robust $\mathscr{H}_{\infty}$ condition since the closedloop system is linear)

$$
\left\|\mathscr{F}_{u}\left(\hat{T}_{c l}(s), \Delta_{u}\right)\right\|_{\infty} \leq \frac{1}{\gamma} \forall \Delta_{u} \in \Delta_{u}
$$

Using the definition of $\mathscr{F}_{u}()$ and the factorization in (22), the above condition implies,

$$
\left\|W(s) \mathscr{F}_{u}\left(T_{c l}(s), \Delta_{u}\right) W^{-1}(s)\right\|_{\infty} \leq \frac{1}{\gamma} \forall \Delta_{u} \in \Delta_{u}
$$


As with the nominal case, the optimization problem will involve a bisection algorithm on $\frac{1}{\gamma^{2}}$ and search for multipliers $\Pi(j \omega)$, $\Pi_{11}(j \omega), \Pi_{12}(j \omega)$ and $\Pi_{22}(j \omega)$ to satisfy the frequencydomain inequality in (16).

\section{DEMONSTRATION OF THE STABILITY MARGIN ANALYSIS}

In this section we will demonstrate an application of the stability margins analysis for the flight control system of an aircraft model that has both rigid and flexible body dynamics. Initially, we will evaluate the bound on $b_{P C}$ assuming zero parametric uncertainty and for this purpose we use a linearized closed-loop system. The closed-loop system is severed at the elevator input of the aircraft and we obtain the loop transfer function as $L(s)$. Next we define $P_{o}(s)=L(s)$ and $C_{o}=1$ and form the closedloop transfer matrix $T_{c l}(s)$ as

$$
T_{c l}(s)=\left[\begin{array}{c}
P_{o} \\
I
\end{array}\right]\left(I-C_{o} P_{o}\right)^{-1}\left[\begin{array}{ll}
-C_{o} & I
\end{array}\right] .
$$

The closed-loop transfer matrix is formed using state-space manipulations of the state-space representation of $L(s)$ in order to avoid duplicating the number of states of $L(s)$. The closedloop transfer matrix $T_{c l}(s)$ has 190 states due to the presence of a large number of flexible modes, sensor and actuator dynamics.

The calculation of the lower bound on $b_{P C}$ was performed using IQC analysis and it was found that $b_{P C}>0.43$ using constant multipliers. The IQC analysis was performed on a reduced version of $T_{c l}(s)$ since otherwise the analysis resulted in infeasible problems due to numerical problems. The model reduction was performed using balanced reduction and states with Hankel singular values less than $2.2 \times 10^{-16}$ were discarded. This resulted in a reduced-order, closed-loop transfer matrix with 90 states. The software used for the calculation of the lower bound were the IQC-toolbox [7] with the semidefinite program solver SDPT3 [13]. The computation time for the lower bound was reduced substantially (by a factor of 8 ) with the use of the dedicated solver for KYP problems KYPD ([15]).

The lower bound on $b_{P C}$ guarantees that the Nichols diagram of $L(s)$ will not enter the ellipse given by,

$$
\left\{\frac{(1+\delta)^{2}}{1-\beta^{2}}:|\delta| \leq \beta=0.43\right\}
$$

This is shown to be the case in Fig. 6 where the ellipse is plotted in red and the Nichols diagram of $L(s)$ is plotted in blue. Note that the ellipse guarantees a phase margin of at least $51^{\circ}$ and a gain margin of at least $8 \mathrm{~dB}$. The actual phase margin is $93^{\circ}$ and the actual gain margin is $8.53 \mathrm{~dB}$, therefore the phase margin estimate suggested by the ellipse is fairly conservative but not the gain margin estimate.

\section{DEMONSTRATION OF THE PERTURBED STABILITY MARGIN ANALYSIS}

In this section we will demonstrate an application of the perturbed stability margin analysis for the flight control system of a longitudinal aircraft model that has both rigid and flexible body dynamics. The closed-loop system has 14 states and the $\Delta$-block has one parameter with multiplicity 16 . The uncertain parameter corresponds to the level of fullness of the trim tanks of the aircraft and it is normalized so that it varies between -1 and 1 . We investigate the perturbed stability margins for the elevator channel.
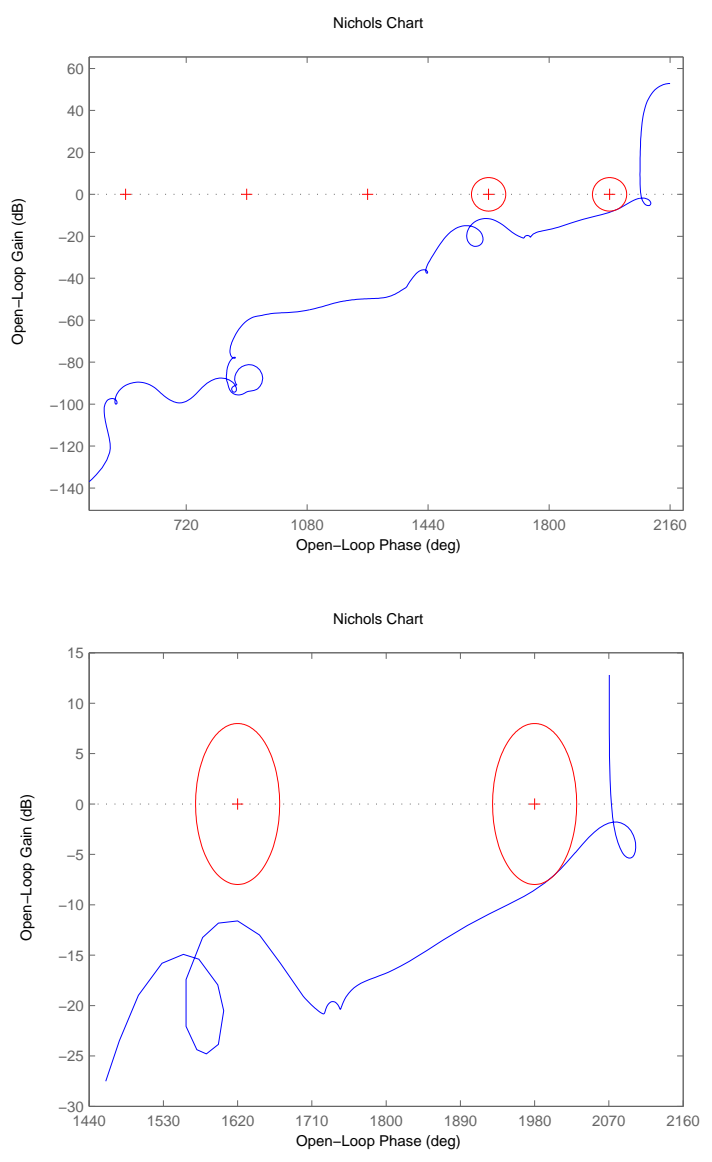

Fig. 6. The Nichols diagram of $L(s)$ and the ellipse.

We evaluate the lower bound on the perturbed $b_{P C}$ as a function of the uncertain parameter size. We start with zero uncertainty $\varepsilon=0$, and let the size of the uncertainty increase to its maximum value $\varepsilon=1$. The lower bound on $b_{P C}(\varepsilon)$ is plotted in Fig. 7 as a function of $\varepsilon$. We see that the controller guarantees adequate stability margins with respect to variations in the trim tank fullness since the worst-case $b_{P C}>0.607$.

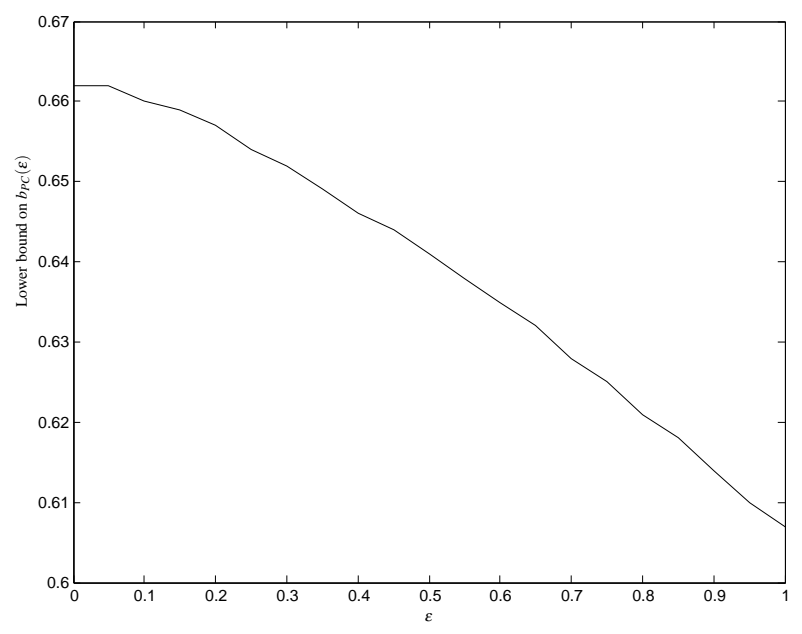

Fig. 7. $b_{P C}(\varepsilon)$ as a function of $\varepsilon$.

In order to establish the conservativeness of the lower bound calculation, we plot the Nichols diagrams for the perturbed loop transfer functions that correspond to the extreme values of the 
uncertain parameter. This is seen in Fig. 8. We also plot the loop transfer function that corresponds to zero uncertainty in green and the ellipse suggested by the lower bound $b_{P C}>0.607$. We see that the lower bound suggested by the perturbed $b_{P C}$ is not very conservative in the sense that it is not very far from the perturbed Nichols plots. Furthermore, the gain margin estimate suggested by the lower bound on the perturbed $b_{P C}$ is also not conservative.

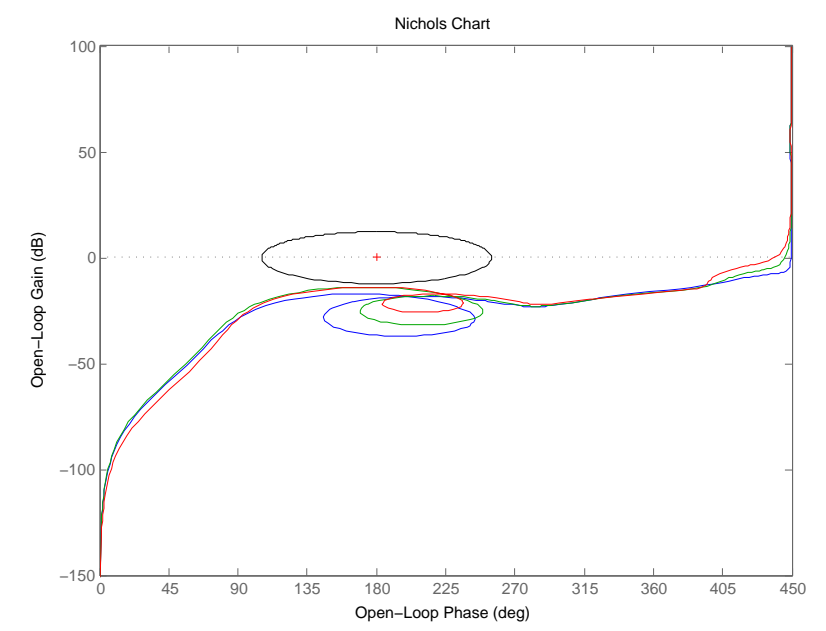

Fig. 8. Nichols plots for the loop transfer functions that correspond to zero uncertainty (green) and to the extreme values of the uncertain parameter (blue, red) along with the ellipse suggested by the lower bound on the worst-case perturbed $b_{P C}$.

The computations for the lower bound on the perturbed $b_{P C}$ were done using SDPT3 [13], and each iteration in the bisection algorithm for calculating the lower bound took about 2 minutes. It should be mentioned that when computing the lower bound using a dedicated solver for KYP-problems such as KYPD, it actually took a longer time to solve the KYP-LMI problem. This is because most dedicated solvers work at their best when the width of the $B$-matrix is small compared to the number of closed-loop states, which is not the case when the size of the $\Delta$-block is actually bigger than the number of states.

\section{CONCLUSION}

This paper deals with the use of convex optimization in order to examine the classical stability margins of a flight control system. The technique is based on the use of the generalized stability margin $b_{P C}$ which has already been shown to have a connection to an exclusion region in the Nichols plane that the loop transfer function does not enter. A non-conservative lower bound on $b_{P C}$ allows the construction of a larger ellipse that the loop transfer function does not enter and therefore implies the satisfaction of better phase and gain margins. We show in this paper how to calculate the lower bound on $b_{P C}$ by solving a semidefinite program based on IQC theory. We are also able to formulate the problem of calculating a lower bound on the perturbed stability margin with respect to variations in parametric uncertainty. In this way we can investigate the classical stability margins in the presence of parametric uncertainty.

\section{REFERENCES}

[1] Anon. General Specification for Flight Control Systems: Design, Installation and Test of Piloted Aircraft. USAF, 1975. Mil. Spec. MIL-F-9490D.

[2] S. Boyd, L. El Ghaoui, E. Feron, and V. Balakrishnan. Linear Matrix Inequalities in System and Control Theory. Studies in Applied Mathematics. SIAM, 1994.

[3] C. Döll, G Ferreres, and J. F. Magni. $\mu$-tools for flight control robustness assessment. Aerospace Science and Technology, (3):177-189, 1999.

[4] C. Fielding, A. Varga, S. Bennani, and M. Selier, editors. Advanced Techniques for Clearance of Flight Control Laws. LNCIS 283. Springer-Verlag Berlin Heidelberg, 2002.

[5] K. Glover, G. Vinnicombe, and G. Papageorgiou. Guaranteed multi-loop stability margins and the gap metric. In Proceedings of the 39th IEEE Conference on Decision and Control, pages 4084-4085, Sydney, Australia, December 2000. IEEE.

[6] K. S. Gunnarsson, J. Hansson, F. Karlsson, A. Hansson, and R. Wallin. Clearance of flight control laws using linear fractional transformations. In AIAA Guidance, Navigation and Control Conference and Exhibit, number AIAA 2004-4867, Providence, Rhode Island, 16-19 August 2004. AIAA.

[7] U. Jönsson, C-Y. Kao, A. Megretski, and A. Rantzer. A guide to IQC $\beta$ : A MATLAB toolbox for robust stability and performance analysis, 1718.

[8] J.-F. Magni. User manual of the linear fractional representation toolbox: Version 2.0. Technical report, ONERA: Systems Control and Flight Dynamics Department, 2006.

[9] D. C. McFarlane and K. Glover. A loop shaping design procedure using $H_{\infty}$ synthesis. IEEE Transactions on Automatic Control, 37(6):759-769, 1992.

[10] A. Megretski and A. Rantzer. System analysis via integral quadratic constraints. IEEE Transactions on Automatic Control, 42(6):819 - 830, 1997.

[11] C. Papageorgiou and K. Glover. Robustness analysis of nonlinear flight controllers. Journal of Guidance, Control and Dynamics, 28(4):639-648, July-August 2005.

[12] J. Steele and G. Vinnicombe. Using the generalised stability margin for flight clearance. In C. Fielding, A. Varga, S. Bennani, and M. Selier, editors, Advanced Techniques for Clearance of Flight Control Laws, LNCIS 283, chapter 4, pages 57-75. Springer-Verlag Berlin Heidelberg, 2002.

[13] R. H. Tutuncu, K. C. Toh, and M. J. Todd. Solving semidefinite-quadratic-linear programs using SDPT3. Mathematical Programming Ser. B, (95):189-217, 2003.

[14] G. Vinnicombe. Uncertainty and Feedback: $H_{\infty}$ loopshaping and the v-gap metric. Imperial College Press, London, 2000.

[15] Ragnar Wallin and Anders Hansson. KYPD: A solver for semidefinite programs derived from the KalmanYakubovich-Popov lemma. In Proceedings of the IEEE International Symposium on Computer-Aided Control System Design, pages 1 - 6, Taipei, Taiwan, 2004.

[16] K. Zhou, J. C. Doyle, and K. Glover. Robust and Optimal Control. Englewood Cliffs, NJ: Prentice Hall, 1996. 


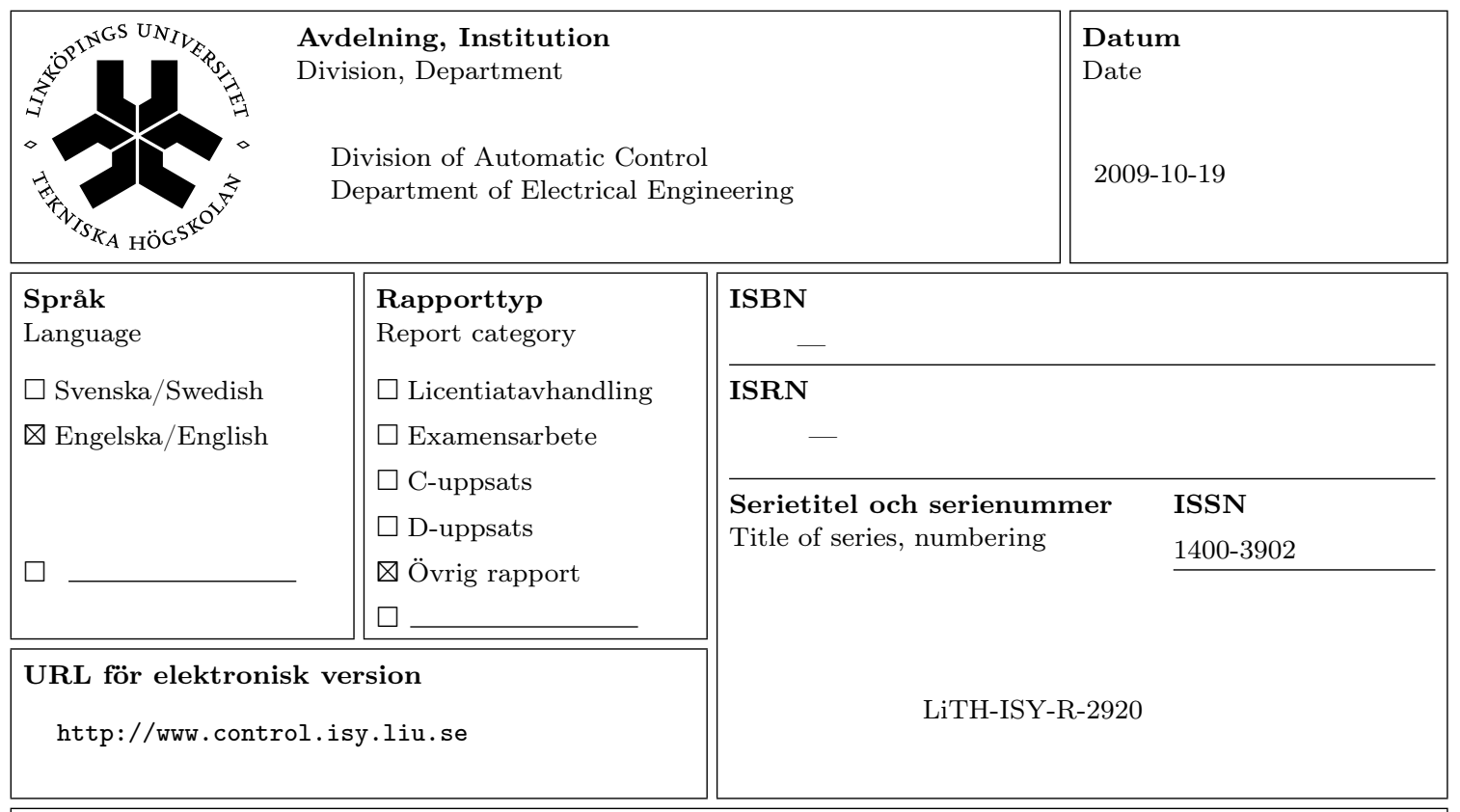

Titel Formulation of the stability margins clearance criterion as a convex optimization problem

Title

Författare Christos Papageorgiou, Rikard Falkeborn, Anders Hansson

Author

Sammanfattning

Abstract

This paper presents the formulation of a flight clearance criterion as a convex optimization problem. The criterion is the stability margins criterion which is specified as an allowable phase and gain margin of a certain loop transfer function. The satisfaction of the criterion amounts to the Nichols plot of the loop transfer function being outside a specified trapezoidal region. It was shown previously that the exclusion condition from an ellipsoidal region is implied by using the generalized stability margin $b_{P C}$ and its calculation was performed frequency-wise by solving a sequence of convex optimization problems. In this paper we formulate the calculation of a lower bound on $b_{P C}$ as a convex optimization problem using Integral Quadratic Constraints (IQCs) and avoid the gridding procedure in the frequency domain. Furthermore, we formulate the problem of obtaining a lower bound on the perturbed stability margin, which is defined as the worst-case $b_{P C}$ over variations in real uncertain parameters. 